\title{
De quelques obstacles rencontrés par les recherches en didactique du français
}

\section{Bertrand Daunay et Yves Reuter}

\section{(2) OpenEdition}

\section{Journals}

\section{Édition électronique}

URL : http://journals.openedition.org/pratiques/1680

DOI : 10.4000/pratiques. 1680

ISSN : 2425-2042

\section{Éditeur}

Centre de recherche sur les médiations (CREM)

\section{Édition imprimée}

Date de publication : 15 juin 2011

Pagination : 9-24

Référence électronique

Bertrand Daunay et Yves Reuter, «De quelques obstacles rencontrés par les recherches en didactique du français », Pratiques [En ligne], 149-150 | 2011, mis en ligne le 16 juin 2014, consulté le 19 avril 2019. URL : http://journals.openedition.org/pratiques/1680; DOI : 10.4000/pratiques.1680

(c) Tous droits réservés 


\title{
De quelques obstacles rencontrés par les recherches en didactique du français
}

\author{
Bertrand Daunay \\ Yves Reuter
}

Théodile-CIREL (ÉA 4354) Université Charles-de-Gaulle - Lille 3

C'est une caractéristique assez courante des disciplines de recherche en sciences humaines de jeter sur elles-mêmes un regard critique, qu'il soit rétrospectif $^{(1)}$ ou qu'il interroge synchroniquement l'état de la discipline ${ }^{(2)}$. Le choix de Pratiques, revue importante dans l'institutionnalisation de la didactique du français, de proposer un état des recherches dans cette discipline, est révélateur d'une certaine maturité de cette dernière, qui a toutes les raisons aujourd'hui de s'interroger sur son devenir : aux raisons épistémologiques, structurelles à toute discipline de recherche, s'ajoutent les facettes (politiques et institutionnelles) d'un contexte qui, pour ne pas lui être favorable (Halté, Petitjean, 2010), lui offre précisément l'occasion d'un retour réflexif sur son cadrage théorique et ses finalités sociales.

Notre contribution à cette entreprise consiste en un retour critique sur certains des obstacles et des difficultés que rencontre aujourd'hui la discipline, retour qui nous semble un moyen efficace pour réfléchir à la pertinence (épistémologique et sociale) des recherches en didactique du français. On peut considérer l'entrée comme pertinente, d'une part parce que les « dysfonctionnements » (Reuter, 2005) que sont les obstacles et les difficultés constituent un bon révélateur d'un «fonctionnement» (théorique ou institutionnel), d'autre part parce que s'intéresser aux obstacles et aux difficultés permet d'éviter de donner prise aux croyances ou aux certitudes décrochées du réel comme aux injonctions non fondées empiriquement.

(1) C'est le cas pour la didactique du français : $c f$. par exemple Schneuwly (1990), Pratiques $\mathrm{n}^{\circ} 137-138$ (2008), La lettre de l'AIRDF n 42 (2008), Enjeux n ${ }^{\circ} 66$ (2006)... Cf. encore le numéro de Repères en préparation ( $\left.{ }^{\circ} 46\right)$.

(2) Cf. notamment Chiss, David, Reuter dir. (1995/2005), Halté (1992), Rosier (2002). 
Quelques précisions liminaires nous paraissent nécessaires, afin de préciser 1'enjeu et les limites de notre approche de la question :

- nous utiliserons le terme recherche au sens large, ce qui inclut les recherches les plus légitimes de laboratoires comme celles qui instituent des chercheurs individuels, comme les thèses ;

- nous privilégierons la didactique du français, mais sans nous interdire de nous référer à d'autres didactiques car, au-delà des différences, il existe des relations entre elles et le devenir des diverses didactiques est en partie lié, comme en témoignent les divers modes de collaboration entre didactiques ou entre didacticiens ${ }^{(3)}$;

- nous nous appuierons sur notre expérience, celle d'acteurs des premières générations de la didactique du français, sur nos responsabilités au sein d'associations de recherche (l'AIRDF particulièrement) et de structures de recherche (Théodile-CIREL), sur nos recherches et nos directions de recherches;

- nous nous centrerons sur le cas français, que nous connaissons le mieux et qui pose des problèmes spécifiques, comme nous essaierons de le montrer dans la suite de cet article.

Le plan que nous proposons (les problèmes institutionnels excédant le champ de la didactique du français ; ceux qui lui sont spécifiques ; les problèmes scientifiques que rencontre la didactique du français) relève d'un découpage certes en partie arbitraire, mais destiné à faciliter le regroupement de questions en vue d'une meilleure lisibilité.

\section{Problèmes institutionnels excédant le champ de la didactique du français}

La didactique du français rencontre des problèmes de nature institutionnelle qui ne sont pas spécifiquement les siens mais qu'elle partage avec d'autres didactiques, voire d'autres disciplines de recherche en éducation ou même, plus largement, en sciences humaines, même si ces problèmes génériques peuvent avoir des effets spécifiques au sein du champ de la didactique du français.

\subsection{Absence de priorité ministérielle pour les recherches en éducation}

Les décisions ministérielles sont rarement appuyées en France sur des recherches spécifiques et moins encore didactiques : ce n'est pas une tradition française, en matière d'éducation, d'identifier des problèmes pour définir une politique de crédits qui permette aux disciplines concernées de tenter d'apporter des réponses fondées théoriquement et empiriquement, dans une temporalité propre

(3) Outre les collaborations ponctuelles importantes entre didacticiens de disciplines différentes ( $c f$. par exemple Margolinas, 2010), on pense ici au programme de didactique comparée (cf. Mercier, Schubauer-Leoni, Sensevy, 2002), qui trouve dans Éducation et didactique un lieu de publication privilégié ; ou aux manifestations et publications qui font dialoguer les didacticiens sur leurs options théoriques ( $c f$. le Dictionnaire des concepts fondamentaux des didactiques, dirigé par Yves Reuter, 2007/2010) ou méthodologiques (cf. les séminaires sur les méthodes de recherche en didactiques : Perrin-Glorian, Reuter dir., 2006 ; Lahanier-Reuter, Roditi dir., 2007 ; Cohen-Azria, Sayac dir. 2009). 
à la recherche, c'est-à-dire longue. On connait plutôt, en France, l'instrumentalisation de la recherche à des fins partisanes, quand tel chercheur ou tel laboratoire est sollicité, dans l'urgence d'une réponse immédiate à des préoccupations politiques conjoncturelles (la violence scolaire, la déscolarisation, l'accueil des handicapés, l'application de programmes...), ou quand telle discipline est appelée à la rescousse d'un discours préconstruit, comme lors de la dernière « querelle de la lecture », instaurée par le ministre Gilles de Robien, qui avait cru bon de faire appel aux sciences cognitives (les « vraies sciences » pour lui ${ }^{(4)}$ ) pour légitimer son exigence d'un prétendu « retour» à la lecture syllabique ${ }^{(5)}$.

Dans tous les cas, les didactiques sont absentes des disciplines jugées légitimes, par les instances décisionnaires, pour penser l'éducation, y compris l'enseignement d'une discipline scolaire ou d'un savoir non disciplinaire. Cela n'était pourtant pas inscrit dans le destin de ces disciplines : au contraire même, s'agissant de la didactique du français ; on peut en effet penser que sa naissance doit beaucoup à la « Rénovation » des années 1970, au sein de 1'IPN-INRDP-INRP ${ }^{(6)}$, qui a tenté d'articuler la recherche et l'élaboration des programmes du primaire : cela a du reste engendré une sorte de tradition de sollicitation de chercheurs dans la rédaction des programmes, pour le primaire mais aussi, dans les années 19902000 , pour le secondaire - avant que les derniers programmes se détournent presque explicitement de toute référence didactique.

Mais il faut se demander si cet épisode fondateur n'a pas finalement nui à la reconnaissance institutionnelle de la discipline, tant la recherche a pu être alors identifiée à l'habillage d'une position militante, position avec laquelle la didactique du français a toujours eu affaire, mais qu'elle a reconstruit différemment tout au long de son développement en prenant soin, ces dernières années, de distinguer ordre de la recherche et ordre du politique, même si certains principes (par exemple l'exigence de lutte contre l'échec scolaire) demeurent fondamentaux, au moins pour certains didacticiens (Daunay, Reuter, 2008). Mais c'est en quelque sorte cette proximité initiale avec une prétendue «pensée 68 » (Ferry, Renaut, 1985) qui peut expliquer la défiance à l'égard de la didactique du français que manifestent tous les tenants d'un conservatisme en matière d'éducation, dont témoignent amplement les manifestes de Sauver les lettres, les plus ardents promoteurs de ce discours conservateur, dont l'activité a été intense, avec l'appui de quelques voix médiatiques, lors des derniers programmes de français pour le lycée.

La participation de certains didacticiens reconnus à la rédaction des programmes du primaire ou du secondaire dans les années 1990-2000 et l'adhésion sans réserve à ces programmes manifestée dans certains écrits didactiques ont contribué à alimenter le discours de dénigrement de la didactique du français, sans argumentation théorique solide mais avec une féroce mauvaise foi doublée d'une efficacité rhétorique, qui est le propre des lobbies idéologiques ( $c f$. Daunay, 2003 ; 2006 ; 2007). Mais il est vrai que certains didacticiens du français, de leur

(4) Libération, 23 février 2006.

(5) Il faut noter que si les scientifiques ainsi indument convoqués dans le discours ministériel ont finalement réagi pour dénoncer l'usage déformé de leurs recherches, c'est assez tardivement, et sans beaucoup d'influence sur le développement du même discours, mais aussi, il faut le noter (et le regretter) sans référence aux recherches didactiques sur la question.

(6) Dénominations successives de l'Institut datant respectivement de 1956, 1970 et 1976. 
côté, n'ont pas toujours su éviter le piège de l'adhésion à des principes d'enseignement qui pouvaient passer pour des mots d'ordre idéologiques.

Toujours est-il qu'aucun programme de recherche conséquent n'a sollicité des laboratoires de recherche en didactique - autonomes de l'institution scolaire - pour à la fois concevoir et évaluer les programmes et leurs possibles effets sur les enseignements et les apprentissages.

\subsection{Durée et mode d'évaluation des recherches}

On évoquait à l'instant les temporalités propres à la recherche, qui ne correspondent pas à celles des injonctions politico-institutionnelles. Cela renvoie à une politique générale de la recherche, qui fait de plus en plus dépendre les financements des laboratoires - et leur reconnaissance institutionnelle - de projets de courte durée. La pérennité des financements - que pouvaient naguère nous envier les chercheurs étrangers - était sans doute plus adaptée à des recherches sur un temps long, qui évitent de simplement suivre l'air du temps ${ }^{(7)}$. Les « expérimentations » des nouveaux dispositifs (mode d'organisation des apprentissages, contenus d'enseignement disciplinaires ou non disciplinaires, structures pédagogiques, etc.), la plupart du temps conçues et pilotées par l'Inspection générale, fussent-elles (parfois, mais rarement) accompagnées par des chercheurs statutaires, sont réalisées dans des temps courts qui empêchent de prendre la mesure de leur efficacité réelle sur le long terme, mais visent plutôt une justification conjoncturelle. L'expérimentation du Plan de rénovation, évoquée plus haut, demeure, à cet égard encore, une exception dans l'histoire de nos disciplines.

La limitation drastique de la durée des thèses, qui s'impose désormais en France, est un autre indice d'une conception étriquée de la recherche : elle interdit les approches longitudinales, pourtant fondamentales pour l'étude des effets, en termes d'apprentissages, des dispositifs d'enseignement. Cette limitation, particulièrement problématique pour les doctorants-enseignants, dont la présence est pourtant essentielle au champ des didactiques, rendrait par exemple impossibles aujourd'hui des thèses comme celles de Francis Ruellan (2000; $f$. Reuter dir., 2005) ou de Martine Fialip-Baratte (2007).

Les choix actuels en matière d'évaluation de la recherche ne sont pas davantage une aide aux recherches en éducation et plus spécifiquement en didactiques. L'exigence de la publication en anglais, qui ne correspond pas exactement à la pente naturelle d'une didactique du français (langue maternelle et/ou première et/ou de scolarisation), la surévaluation des dimensions quantitatives, qui n'est pas l'approche principale de la plupart des didactiques ${ }^{(8)}$, le privilège accordé aux découvertes « scientifiques » ou aux «brevets », qui ne correspond pas aux principes des didactiques, sont autant de signes d'une absence de prise en compte des spécificités de notre champ.

Il faut ajouter à cela, mais c'est sans doute lié, que les didacticiens sont rarement sollicités pour l'expertise scientifique dans les structures institutionnelles,

(7) Une recherche comme celle qu'a menée l'équipe Théodile sur l'école Freinet (Reuter dir., 2007b) serait rendue plus difficile aujourd'hui.

(8) Le problème en l'occurrence tient à la manière dont les approches dites « quantitatives " sont opposées de manière souvent fruste à des approches qui seraient « qualitatives ». 
que les recherches en didactiques sont souvent évaluées par des experts d'autres disciplines (à l'ANR notamment), que les laboratoires de didactiques relevant des sciences de l'éducation étaient encore récemment évalués dans une commission de l'AERES dont le délégué scientifique relevait de la psychologie ${ }^{(9)}$, que les didactiques n'ont, pas plus que les sciences de l'éducation, de section spécifique au CNRS.

\subsection{Instabilité de la formation des maîtres}

La remise en cause des Instituts universitaires de formation des maîtres (IUFM) en France engendre une insécurité non seulement pour nombre d'enseignants-chercheurs, dont les missions sont à redéfinir, mais aussi pour les didactiques : outre que la disparition de l'autonomie des IUFM tend à diminuer le nombre de postes qui étaient attribués à ces disciplines, les nouveaux masters professionnels, surtout dans le second degré, font craindre une diminution de la part des didactiques dans la formation des enseignants et la diminution des postes de didactique.

De fait, les diverses réformes récentes du recrutement et de la formation des enseignants se sont effectuées sur des bases qui étaient clairement une mise en cause des disciplines de recherche destinée à l'appréhension des phénomènes éducatifs. Ainsi, le choix des universités de rattachement des IUFM ne s'est pas effectué sur la base d'une rationalisation des ressources en matière d'éducation, quand, dans plusieurs académies, l'IUFM s'est vu rattaché à une université totalement privée de laboratoire ou de département de sciences de l'éducation, quand bien même une autre université de la même académie disposait de telles ressources. De même, la «mastérisation » de la formation des enseignants n'a pas été guidée par la volonté de concevoir une exploitation des compétences (en enseignement universitaire ou en recherche) des enseignants-chercheurs spécialistes des didactiques et des questions d'enseignement : c'est bien sûr une litote, dans la mesure où, particulièrement dans le secondaire général, c'est une « restauration » des disciplines académiques qui était visée. Cela entre bien en concordance avec la «culture du mépris » que développent parfois les mêmes disciplines académiques à l'égard des didactiques et des recherches en éducation en général ${ }^{(10)}$.

\section{Problèmes institutionnels spécifiques au champ de la didactique du français}

Si la didactique du français partage certains problèmes de nature institutionnelle avec d'autres didactiques, elle en connaît qui lui sont propres, même si parfois la frontière est ténue entre le générique et le spécifique en la matière : ce pourrait être, du reste, un travail intéressant de didactique comparée que de faire la part de l'un et de l'autre...

(9) La donne a changé seulement en 2010, où a été nommé un délégué scientifique issu des sciences de l'éducation pour organiser les évaluations des laboratoires de sciences de l'éducation.

(10) Pour une description critique des ressorts de cette culture du mépris, $c f$. Daunay (2007). 


\subsection{Une discipline d'une grande « sensibilité sociale » (11)}

La didactique du français a, par nature, des objets de recherche qui renvoient à des pratiques socialement partagées et sensibles : la langue, la lecture, 1'écriture, la littérature, par exemple, sont des objets de forts enjeux identitaires (personnels aussi bien que communautaires) et de pratiques ou de représentations communément partagées, dont chacun, légitimement, se sent investi du droit de parler et de parler de son enseignement ( $c f$. Reuter, 2004). C'est ce qui explique en partie la difficulté à supposer, pour nombre de médias ou pour le grand public, qu'il y ait des « spécialistes » de l'enseignement du français : l'orthographe, la grammaire, la lecture, l'écriture, la littérature, etc., sont l'objet de discours nombreux qui prolifèrent sur un fond de déni de toute spécialité scientifique, didactique notamment.

Ce déni est porté à son paroxysme - moins légitimement à coup sûr - par quelques « experts » médiatiquement désignés, dont la parole se caractérise par un rejet de tout discours scientifique en matière d'enseignement du français, rejet proportionnel à l'ignorance de ce discours : la dernière illustration en est la querelle née à l'occasion de la parution des programmes de français pour le lycée en France, en 2001, qui a donné lieu à une frénésie éditoriale ${ }^{(12)}$. Il est frappant en effet de voir, dans les textes produits à cette occasion, comment les didacticiens peuvent être pourfendus sans jamais une référence explicite à un seul didacticien...

L'absence de prise en compte des discours de l'adversaire supposé explique la possibilité de fantasmer sur un discours ennemi qui soit homogène : « les pédagogues », « les didacticiens » parlent - et imposent leurs paroles, par une vertu incantatoire qu'ils tiennent de leur supposé pouvoir... Il ne vient jamais à l'esprit (en tout cas à la plume) des opposants à la réforme qu'au sein même du champ didactique, les critiques ont été parfois rudes ; mais surtout, qu'au-delà de l'arrivée de tel ou tel nouveau programme, il existe en didactique des tendances voire des conflits théoriques; que les maux qu'ils signalent ont été déjà décrits depuis longtemps par des auteurs de ce champ ; que même certaines de leurs propositions ont déjà été préconisées et défendues théoriquement.

Avatar de la «culture du mépris » évoquée plus haut, qui dévalorise les disciplines touchant à l'enseignement, ce déni d'une approche didactique de l'enseignement rencontre l'expression ordinaire d'un « bon sens » suspectant assez naturellement toute réflexion théorique qui en interrogerait les présupposés, celui par exemple qui considère comme immuable l'enseignement initial de la lecture, de la grammaire et de la littérature, quand est décerné un label d'éternité à la méthode Boscher, au Bled ou au Lagarde et Michard ${ }^{(13)} \ldots$

(11) Reuter (2008).

(12) Voir le corpus partiel que propose Daunay (2003) et le corpus plus complet de Plane (2001), qui en fait une analyse critique ( $c f$. aussi Coget, 2003 ; Paveau 2001 ; Petitjean 2001).

(13) Ces trois exemples sont emblématiques de méthodes dites aujourd'hui «traditionnelles » (notamment parce que l'on en a oublié le caractère parfois novateur au départ...) concernant l'apprentissage initial de la lecture, l'entrainement grammatical (primaire, collège), la littérature (lycée), et souvent citées en contre-exemples des méthodes supposées moins efficaces des «nouvelles » pédagogies, ce qui peut du reste expliquer leur succès éditorial, encore aujourd'hui. 
Ce «bon sens », du reste, n'a rien d'aberrant et n'est pas étranger à une certaine culture scolaire forgée par la tradition de l'enseignement du français, dont André Chervel (2006) a bien montré la capacité de permanence, de reproduction et d'adaptabilité : au mieux, les réformes successives n'effacent que partiellement et sur un long terme les finalités et les pratiques anciennes. Certains didacticiens du français, épris d'innovation et passant parfois bien vite de l'exploration de possibles à la préconisation, ont sans doute mal mesuré la nécessité de la prise en compte de la tradition scolaire pour rendre son discours théorique plus vraisemblable au regard des pratiques effectives et des possibilités d'évolution du système éducatif.

\subsection{L'inscription dans divers cursus disciplinaires}

La didactique du français en France est inscrite dans des cursus de formation divers : sciences de l'éducation, sciences du langage, littérature, information et communication, etc., ce qui correspond du reste à l'affiliation universitaire des didacticiens, qui peuvent relever de diverses sections du Conseil national des universités (CNU). Cela entraîne évidemment une difficile lisibilité institutionnelle de la didactique du français comme discipline de recherche et de formation universitaire (cette dernière étant par ailleurs assez courte : la formation didactique commence généralement au niveau de la troisième année de licence).

L'articulation de la formation (initiale et doctorale) et de la recherche à des disciplines diverses peut être en soi une richesse, puisque cela peut diversifier les formes d'ancrage des questions didactiques et le dialogue entre disciplines diverses, mais peut se révéler une faiblesse quand les approches didactiques sont identifiées comme relevant de paradigmes non didactiques ou comme une extension d'une discipline de référence. Cela nuit en effet à la constitution d'un champ théorique autonome et concourt à la constitution d'une sorte de kaléidoscope où la didactique du français peut être perçue comme la fédération, hasardeuse épistémologiquement, de didactiques spécifiques - de la langue, des discours, des textes, de la littérature... Autrement dit, l'inscription de la formation en didactique dans des disciplines académiques diverses peut concourir à la constitution verticale de sous-champs théoriques divers (linguistique $\rightarrow$ didactique de la langue ; littérature $\rightarrow$ didactique de la littérature, etc.) ; inversement, la constitution horizontale d'un champ autonome serait favorisée par l'appartenance de la didactique aux sciences de l'éducation, mais cela aurait pour conséquence une coupure avec les disciplines académiques contributoires, ce qui n'est pas sans poser d'autres problèmes... Car, finalement, où peut réellement se construire une perspective intégrative d'une didactique conçue comme la discipline où se construisent les problématiques d'enseignement et d'apprentissage des compétences langagières (Halté, 1992)?

Cette impression d'un kaléidoscope théorique et institutionnel s'accentue encore par la cohabitation (plus que par le dialogue épistémologique) d'une didactique du français langue première d'un côté et, de l'autre d'une didactique du français langue étrangère (pour nommer ainsi une discipline qui ne se résume pas à la langue étrangère mais intègre les problématiques de la langue seconde, de scolarisation, de spécialité, etc.). 


\subsection{Tension entre recherche et recommandation}

Pour les raisons évoquées à l'instant, le risque de l'applicationnisme n'est pas totalement écarté de notre champ, la didactique passant parfois, y compris chez certains de ses spécialistes, pour l'application de savoirs élaborés dans d'autres disciplines. On peut identifier un double applicationnisme :

- théorique, quand il y a relation descendante entre une approche théorique « académique » et une application théorique didactique (c'est le cas, par exemple, lorsque tel concept d'une théorie du texte ou de la littérature est sorti de son champ initial pour être introduit dans le champ didactique sans travail de recontextualisation théorique);

- pratique, quand s'opère une relation descendante entre une approche théorique et une approche technologique (par exemple l'application de savoirs théoriques sur la langue dans l'enseignement grammatical). Ce dernier applicationnisme est fréquemment illustré par l'utilisation du syntagme « approches théoriques et didactiques », sous la plume de spécialistes d'autres disciplines mais parfois aussi sous celles de didacticiens, syntagme qui donne à supposer un partage entre une dimension théorique relevant de disciplines académiques non didactiques et une dimension applicative dévolue à la didactique.

L'applicationnisme révèle une tension, qui n'est pas propre à la didactique mais concerne plus généralement toutes les composantes du champ de recherche en éducation ( $c f$. Daunay, Reuter, 2008), entre l'espace de la recherche (qui veut décrire, comprendre, expliquer des phénomènes éducatifs spécifiques que vise à appréhender la didactique du français) et celui de la recommandation - qui suppose possible de déduire un programme d'action d'un programme scientifique mais néglige que la réduction scientifique de l'objet traité ne correspond pas exactement aux exigences de l'action effective ( $c f$. Bucheton, Soulé, 2009).

Est-ce aux chercheurs de participer à la conception de programmes, de manuels, d'ouvrages de préparation aux concours, d'ouvrages de formation? L'expertise qui leur est reconnue dans ces occasions est certes une forme importante de reconnaissance institutionnelle et éditoriale, mais elle contribue à engendrer une confusion entre les instances de recherche et de recommandation, qui fragilise la légitimité d'un champ de recherche ${ }^{(14)}$. Si la pensée de l'intervention est constitutive de la didactique du français comme champ de recherche (voir Bronckart, 2001 ; Martinand, 1987 ; Halté, 1992 sur la notion d'implication ; Reuter, 1995/2005 sur la notion d'horizon praxéologique), il manque une réflexion épistémologique d'ampleur collective, qui serait nécessaire à la clarification de son programme scientifique, sur les conditions de l'articulation des recherches théoriques au champ des pratiques, qui ne saurait, sous peine d'être elle-même athéorique, se résoudre en une simple application (doublée d'une subordination) de l'un à l'autre.

Si, dit Marc Bru (1998, p. 54), « les obstacles de l'orientation prescriptive ne condamnent pas les sciences de l'éducation à abandonner toute mise en relation

(14) Sur la distinction entre espaces de prescriptions, de recommandations, de représentations, de pratiques, de recherches, voir Reuter (2007), pp. 69-70. 
de recherches et des pratiques », il reste, ajoute-t-il, «à modéliser ces relations sans les réduire à une visée applicationniste ou à une version idéalisée, étrangères à ce que sont les pratiques en situation. »

\subsection{Problèmes de légitimité et comportement évaluatif}

Les problèmes de légitimité de la discipline, que peuvent intérioriser certains de ses acteurs, font observer une oscillation somme toute assez classique entre laxisme de soutien aux recherches internes au champ et soumission exacerbée à des normes externes. Du côté de cette dernière, il est curieux de voir comment se construit progressivement un discours de dénigrement des caractéristiques principales de notre champ au nom de normes héritées d'autres traditions scientifiques : c'est ce qui explique par exemple le peu de cas fait, dans ce discours - qui gagne institutionnellement du terrain - de la diffusion, en français, des recherches dans des espaces de publication professionnels, non cotés scientifiquement, mais qui font partie intégrante du travail scientifique; ou encore le primat donné à la publication dans des revues, jugées plus « scientifiques » que la publication d'un ouvrage.

A l'inverse de ce discours, s'observent aussi des pratiques d'évaluation parfois peu sévères de productions internes au champ. C'est, de fait, le cas dans la majeure partie des disciplines, y compris chez celles dont certains représentants sont toujours prêts à donner des leçons aux didactiques, mais cela ne saurait constituer une quelconque excuse scientifique même si cela interroge l'articulation entre science et institutions, dont on sait à quel point elle est cruciale. Cela s'observe tant dans les évaluations de certains articles dans les revues que dans les jurys de thèses. Il arrive que l'on oublie que les thèses, par exemple, doivent être contributoires à un champ de recherche et ne relèvent pas de l'essai personnel ou encore qu'elles doivent tenir compte de l'histoire des recherches dans le domaine. Il est ainsi parfois étonnant de constater que telle thèse fait débuter la réflexion sur l'écriture et sur son enseignement aux modèles cognitivistes américains en oubliant les travaux d'Halté ou de Petitjean par exemple, ou d'observer que telle thèse insiste sur la nouveauté de la recherche sur les pratiques d'enseignement du français en oubliant, entre autres, le travail pionnier de Frank Marchand (1971).

\section{Problèmes scientifiques}

Les dimensions institutionnelles rejaillissent sur le programme scientifique des didactiques, notamment parce qu'il n'y a pas eu encore de théorisation suffisante des problèmes que la situation institutionnelle et les structurations mêmes des didactiques engendrent.

\subsection{La relation avec les « disciplines-mères"}

L'inscription dans divers lieux institutionnels de la didactique comme champ de recherche, dont on a évoqué plus haut (2.2) certaines conséquences disciplinaires, peut nuire également à la clarté du programme scientifique de la didacti- 
que du français quand ses objets et ses méthodes sont comme le calque de ceux des disciplines «d'accueil », au risque d'une difficulté à construire la spécificité de la discipline de recherche qu'est la didactique du français. On sait du reste le rôle décisif d'une certaine forme d'applicationnisme dans l'émergence de la didactique du français, quand la linguistique imposait sa marque à de nombreuses autres disciplines et leur permettait de construire des méthodes et des problématiques nouvelles dans leur propre champ de recherche. Mais cette impulsion peut poser problème pour la construction d'une spécificité théorique de la discipline (toujours) nouvelle qu'est la didactique : par exemple, il y a lieu de se demander si certaines descriptions des pratiques enseignantes ne sont pas davantage linguistiques que didactiques ou, sous une autre forme, ce qui fait la spécificité d'une description didactique des pratiques enseignantes, des interactions dans les classes...

Il en est de même avec les études littéraires, dont le renouvellement des années 1960 a été un autre moteur de la constitution du champ didactique, mais dont l'influence peut devenir un frein désormais, quand l'approche didactique croit pouvoir se limiter à des conceptions nées de ces théories, sans reconfiguration didactique. Par exemple, combien de discours sur la "singularité » ou l' "identité » de l'appréhension du texte littéraire seraient évités (ou, du reste, enrichis), s'ils étaient mis en dialogue avec les recherches sociolinguistiques ou sociologiques sur l'appréhension de la langue (par exemple Bourdieu, 1982 ; Labov, 1976 ; Gardin, Marcellesi, Greco, 1980) ou avec les recherches de sociologie culturelle ( $c f$. Pratiques n 32, 1981 ; Rosier, Dupont, Reuter, 1988/2000)?

On voit bien le double problème que pose la soumission à ce qu'il est convenu parfois d'appeler les «disciplines-mères » : outre qu'elle peut encore se traduire par la reprise des vogues théoriques et les tentations applicationnistes (de la linguistique, de la narratologie, des typologies textuelles, etc.) ${ }^{(15)}$, elle peut interdire la recherche d'une intégration des problématiques du "français » comme matière d'enseignement.

Supposer des « disciplines-mères », c'est entériner la dualité que dénonçait Jean-Paul Bronckart (2001, p. 136) :

Entre les sciences humaines fondamentales et le domaine de l'éducation, se sont établis les rapports hiérarchiques et descendants de l'applicationnisme : les données scientifiques étaient injectées dans le champ pratique, la plupart du temps sans réelle prise en compte des multiples paramètres qui régissent ce dernier.

C'est précisément ce que les fondateurs de la didactique du français voulaient éviter, en l'instaurant comme possible discipline de référence du français (matière d'enseignement) pour en déterminer la « matrice disciplinaire » : c'est ce qu'a toujours souhaité particulièrement Jean-François Halté (1992;1995/2005) qui, constatant la difficulté du « français-matière » à « boucler son référentiel savant (mais aussi ses référentiels culturels et ses référentiels pratiques) », suggérait « de construire cette matrice à partir de la matière, à partir des finalités et des

(15) Dont on voit les traces, selon la typologie des recherches didactiques de Gagné, Lazure, Sprenger-Charolles, Ropé (1989, p. 58), dans 1'importance de l'application théorique d'éléments disciplinaires. Sur ce point, concernant la didactique de la littérature, $c f$. Daunay, Dufays (2007, p. 12). 
objectifs de l'enseignement et non dans les disciplines de référence » (2001, p. 17). Si une telle option est possible, il échoit à la didactique du français de penser la matrice de la discipline ( $c f$. Daunay, Reuter, 2008).

\subsection{Les débats avec les disciplines contributoires}

Considérer la didactique comme discipline autonome suppose de constituer les « disciplines-mères " en disciplines contributoires, ce qui veut dire dialoguer avec elles et leur emprunter, mais en les reconfigurant, leurs méthodes, leurs concepts, leurs problématiques ${ }^{(16)}$. Il serait nécessaire que la didactique du français identifie plus clairement (et plus collectivement) une sorte de cartographie des disciplines qui lui sont contributoires : outre les disciplines identifiées comme sources de savoirs possiblement institués savoirs de référence des savoirs scolaires, peuvent avoir ce statut de disciplines contributoires les autres didactiques mais aussi la pédagogie et, dans un autre cercle, toutes les théories qui, d'une manière ou d'une autre, interrogent à leur manière les phénomènes que la didactique du français veut appréhender, au premier rang desquelles (parce que traditionnellement sollicitées par la didactique du français) la sociologie et la psychologie.

L'élaboration de cette cartographie dessinerait un ambitieux programme de reconfiguration interne à la didactique du français des savoirs issus de ces disciplines, sans nécessairement nécessiter une approche de haute généralité théorique, mais comme en acte, dans toute recherche didactique, qui se donnerait comme projet d'identifier les références convoquées et leur reconfiguration effective. Or il faut reconnaitre là une faiblesse de nombre de travaux en didactique du français : si les emprunts sont fréquents avec les disciplines contributoires, les débats théoriques avec elles sont souvent insuffisants ${ }^{(17)}$. On peut penser par exemple à certains emprunts aux disciplines contributoires qui ne sont pas toujours pensés dans un cadre théorique didactique propre : la question du travail enseignant est traitée parfois dans l'oubli des travaux antérieurs ( $c f$. Delcambre, Reuter, 2006) ou sans véritable interrogation des conditions d'emprunts aux autres disciplines (ergonomie, psychologie du travail...) ; la notion de " sujet », convoquée assez régulièrement en didactique, n'est pas toujours pensée dans un cadre théorique didactique, ce qui amène à voir dans le sujet décrit davantage un sujet sociologique, psychologique ou pédagogique qu'un «sujet didactique » ( $c f$. Daunay, à paraître); la notion de « métalinguistique » est souvent sollicitée en didactique du français mais sans que soit toujours pensées les différences de construction de la notion selon qu'elle est construite, par exemple, en linguistique, en psycholinguistique, en sociologie et sans que soient explicitées les modalités d'intégration en didactique des emprunts de cette notion à d'autres disciplines. On pourrait également interroger comment, en didactique, sont mis en réseau des notions d'origines diverses : par exemple, les notions de « forme

(16) Sur les relations entre les didactiques et les autres disciplines, voir, par exemple, Reuter dir. (2007), pp. 79-84.

(17) Ce n'est pas là une faiblesse de la discipline toute entière, car elle présente aussi de nombreux exemples de dialogues avec d'autres disciplines, y compris les autres didactiques. Les autres disciplines (dont les didactiques) sont loin de toutes présenter ces mêmes qualités de dialogue... 
scolaire » et de «transposition didactique» sont parfois mises en relation d'une manière qui néglige leur socle d'origine, essentiellement critique quant à l'école (chez Verret, 1975 ou Vincent, 1980); les articulations entre les théories de Bakhtine, de Goody, de Vygotski sont parfois effectuées dans une forme de « syncrétisme» théorique qui passe sous silence les possibles frictions entre les cadres théoriques de ces auteurs ( $c f$. Reuter, 2006).

\subsection{Le travail métathéorique}

La capacité de dialogue avec des disciplines va de pair avec une clarification conceptuelle et méthodologique interne. Si les annonces de « rupture épistémologique » sont assez nombreuses et régulières, elles ne disent rien de la capacité de la didactique du français à fonder son cadre théorique sur des concepts spécifiques éprouvés collectivement.

Il est frappant, par exemple, de voir le peu de concepts propres à la didactique du français ayant fait fortune au-delà des recherches de leurs inventeurs ${ }^{(18)}$ ou encore la faible spécificité des méthodes propres aux didactiques du français, malgré certaines avancées récentes (notamment dans l'analyse de l'activité enseignante), mais trop peu encore éprouvées par des croisements critiques entre elles.

La didactique du français, de ce point de vue, fonctionne encore comme une discipline neuve, où chacun teste ses concepts et ses méthodes, sans la nécessaire confrontation systématique, qui fait le propre des théories avancées. Ce déficit s'observe notamment dans une question somme toute banale et pourtant redoutable, celle de l'appréhension des "performances » des élèves et de l' «efficacité » des dispositifs didactiques, question ${ }^{(19)}$ qui, par nature, est consubstantielle à la didactique du français, si on adhère à sa dimension praxéologique : or rares sont les réflexions métathéoriques qui ne soient pas ad hoc sur une telle question, ce qui amène à interroger la légitimité même des propositions d'action qui pourtant, elles, fleurissent sans beaucoup de résistance...

\subsection{Perte de mémoire et des priorités}

Tous les problèmes évoqués ci-dessus se condensent dans une difficulté de la discipline à se fonder sur un dialogue constructif avec l'existant : c'est un constat souvent fait que la culture de la capitalisation scientifique n'est pas encore très ancrée dans les pratiques des chercheurs : il n'est pas rare de relire pour la énième fois ce qui pourtant se présente comme une nouveauté, sans référence à des écrits anciens qui avaient pourtant soit traité la question, soit déjà anticipé les objections que l'on peut faire à telle ou telle proposition théorique ou pratique.

Là encore, on peut avancer, au risque d'un effet de discours nostalgique, que les fondateurs de la discipline avaient pensé la question, grâce au travail consi-

(18) On peut en juger dans le Dictionnaire des concepts fondamentaux des didactiques (Reuter éd., 2007a). On pourrait d'ailleurs dire sensiblement la même chose à propos du nombre de concepts d'autres didactiques, repris à d'autres champs théoriques et finalement de peu d'extension.

(19) Formulée notamment dans Daunay (2008) et Reuter (à paraître). 
dérable réalisé dès les années 1980 par quelques pionniers qui avaient engagé la construction de la banque de données DAF, articulée à un travail métathéorique d'envergure (Gagné, Lazure, Sprenger-Charolles, Ropé, 1989). Mais, de même que les incertitudes pesant sur les missions de l' INRP ne lui ont pas permis de se constituer comme lieu de capitalisation des données de recherche, l'absence de moyens alloués à la banque de données DAF a fait quasiment disparaître le seul outil existant en la matière dans notre discipline.

Par ailleurs, rares sont les notes de synthèse en didactique du français (voire en didactiques) ; et comme les notes de synthèses faites dans les autres disciplines ne mentionnent que très rarement les recherches en didactique du français, le déficit en synthèse des recherches est grand dans notre champ. La tradition des revues thématiques dans le champ de la didactique du français, curieusement, pourrait favoriser cette capitalisation, alors qu' au contraire, elle semble par moments susciter une certaine répétition des mêmes discours, mais sans que se constitue une véritable culture de l'état de l'art pour les objets abordés, ni, en compensation, l'émergence de nouveaux thèmes que des revues non thématiques pourraient permettre.

La perte de mémoire fait courir le risque d'une perte des priorités : on peut parfois se demander, dans certaines présentations de recherches, ce qui reste de la problématique de l'échec scolaire, fondatrice de la didactique du français. Plus généralement, actuellement, quelles réponses spécifiques la discipline apporte-telle à cette question fondamentale, éthiquement, politiquement et socialement ?

\section{Conclusion}

Notre approche critique de quelques obstacles et difficultés que rencontre aujourd'hui la didactique du français n'est pas, en apparence, très productive : nous ne proposons pas de solution concrète, dans un contexte politico-institutionnel qui nous inquiète autant que la plupart des membres de notre communauté...

Il nous faut encore mettre en garde contre deux effets de lecture que ces lignes pourraient susciter et qui seraient le fait d'une maladresse d'expression :

- l'effet « donneurs de leçon »: loin de cette posture, nous nous sentons solidaires des difficultés collectivement rencontrées et nous nous reconnaissons volontiers dans certaines des critiques que nous soumettons à la discussion ;

- l'effet « ruine de la discipline » : à lire ce qui précède, on pourrait supposer que la didactique du français - ou les didactiques - seraient plus en difficultés que d'autres disciplines ou moins aptes qu'elles à les résoudre, alors qu'une même attention critique aux problèmes de ces disciplines aurait toute chance d'aboutir à des conclusions similaires.

Notre ambition (sans doute démesurée), en écrivant ces lignes, est qu'elles aident à dessiner sinon des perspectives d'avenir, au moins des balises pour une action future. La didactique du français a fait la preuve de son inventivité, depuis trois décennies : elle devrait parvenir à surmonter les obstacles quelle rencontre, au prix d'une certaine lucidité sur ses difficultés actuelles. 


\section{Références}

BoURDIEU, Pierre (1982) : Ce que parler veut dire, Paris, Fayard.

BRONCKART, Jean-Paul (2001) : «S'entendre pour agir et agir pour s'entendre », dans Baudouin Jean Michel, Friedrich Janette éd., Théories de l'action et éducation, Bruxelles, De Boeck Université, pp. 133-154.

BRU, Marc (1998) : «Qu'y a-t-il à prouver, quand il s'agit d'éducation ? La validation scientifique des propos et discours sur les pratiques d'enseignement: après les illusions perdues », dans Hadji Charles, Baillé Jacques, éds., Recherche et éducation. Vers une "nouvelle alliance ». La démarche de preuve en 10 questions, Paris-Bruxelles, De Boeck, pp. 45-65.

Bucheton, Dominique, Soulé, Yves (2009) : «Les gestes professionnels et le jeu des postures de l'enseignant dans la classe : un multi agenda de préoccupations enchâssées ", Éducation et Didactique, Vol. 3, n 3, Rennes, PUR, pp. 948.

CHERVEL, André (2006) : Histoire de l'enseignement du français du XVII au XX siècle, Paris, Retz.

CHISs, Jean-Louis, D AVID, Jacques, REUTER, Yves dir. (1995/2005) : Didactique du français. Fondements d'une discipline, Bruxelles, De Boeck.

Coget, Clémence (2003) : «La réaction fait sa révolution », Recherches $n^{\circ} 39$, Écriture d'invention, Lille, ARDPF, pp. 69-79.

COHEN-AZRIA, Cora, SAYAC, Nathalie, dir. (2009) : Questionner l'implicite. Les méthodes de recherche en didactiques (3), Villeneuve d'Ascq, Presses universitaires du Septentrion.

DAUNAY, Bertrand (2003) : «Les discours sur l'écriture d'invention et les frontières de la discipline », Recherches $\mathrm{n}^{\circ} 39$, Écriture d'invention, Lille, ARDPF, 2003-2, pp. 39-68.

- (2006) : «Lecture littéraire et disqualification scolaire », Lidil n ${ }^{\circ} 33$, La Réception des textes littéraires. Une didactique en construction, Grenoble, LIDILEM, pp. 19-35.

- (2007): Invention d'une écriture de recherche en didactique du français, Note de synthèse en vue de l'habilitation à diriger des recherches, Université Charles-de-Gaulle - Lille 3, mai.

— (2008) : Performances et apprentissages disciplinaires », Cahiers Théodile $\mathrm{n}^{\circ}$ 8, Villeneuve d'Ascq, Université Charles de Gaulle - Lille 3, pp. 7-23.

— (à paraître) : "L'enfant, l'élève, l'apprenant en didactique du français ", Recherches en didactiques. Les Cahiers Théodile $n^{\circ} 11$, REDLCT, Lille 3.

DAUNAY, Bertrand, DUFAYS, Jean-Louis (2007) : « Méthodes de recherche en didactique de la littérature », Lettre de l'AIRDF n ${ }^{\circ} 40$, pp. 8-13, Namur.

DAUNAY, Bertrand, REUTER, Yves (2008) : «La didactique du français : évolution, méthodes et enjeux », Pratiques $\mathrm{n}^{\circ}$ 137-138, La didactique du français. Hommage à Jean-François Halté, Metz, CRESEF, pp. 57-78.

DELCAMBRE, Isabelle, REUTER, Yves (2006) : «L'objet enseigné comme objet à construire. Une discussion critique », dans Schneuwly Bernard, ThévenazChristen Thérèse dir., Analyses des objets enseignés. Le cas du français, Bruxelles, De Boeck, pp. 233-246.

DEVElay, Michel (1992) : De l'apprentissage à l'enseignement, Paris, ESF. 
ENJEUX $\mathrm{n}^{\circ} 66$ (2006) : Enseignement et engagement. Hommage à Jean-Maurice Rosier, Namur, CEDOCEF.

FERRY, Luc, RENAUT, Alain (1985) : La Pensée 68. Essai sur l'antihumanisme contemporain, Paris, Gallimard.

FIALIP-B ARATTE, Martine (2007) : La construction du rapport à l'écrit. L'écriture avant l'écriture. Discours d'enfants de maternelle, Paris, L'Harmattan.

GAGNÉ, Gilles, LAZURE, Roger, SPRENGER-CHAROLLES, Liliane, Ropé, Françoise (1989) : Recherches en didactique et acquisition du français langue maternelle, Tome 1, Cadre conceptuel, thésaurus et lexique des mots-clés, Bruxelles, De Boeck-Wesmael ; Paris, Editions Universitaire-INRP ; Montréal, Université de Montréal-PPMF.

GARDIN, Bernard, MARCELlESI, Jean-Baptiste et G.R.E.C.O. de Rouen (1980), Sociolinguistique. Approches, théories, pratiques, Rouen, Presses universitaire de Rouen.

Halté, Anne, Petitjean, André (2010) : «État des recherches en didactique du français. 1. Les pratiques enseignantes », Pratiques $\mathrm{n}^{\circ}$ 145-146, Didactique du français, Metz, CRESEF, pp. 3-7.

HALTÉ, Jean-François (1992) : La didactique du français, Paris, PUF, Que sais-je ?

LA LETTRE DE l'AIRDF $\mathrm{n}^{\circ} 42$ (2008) : La didactique du français : coup d'œil vers le passé. En hommage à Jean-François Halté, Namur, AIRDF.

LABOV, William (1978) : Sociolinguistique, Paris, Minuit.

LAHANIER-REUTER, Dominique, RODITI, Éric dir. (2007) : Questions de temporalité. Les méthodes de recherche en didactiques (2), Villeneuve d'Ascq, Presses universitaires du Septentrion.

MARGOLINAS, Claire (2010) : «Recherches en didactique des mathématiques et du français : par-delà les différences », Pratiques n ${ }^{\circ}$ 145-146, Didactique $d u$ français, Metz, CRESEF, pp. 26-32.

MARTINAND, Jean-Louis (1987) : «Quelques remarques sur les didactiques des disciplines, Les sciences de l'éducation. Pour l'ère nouvelle ${ }^{\circ} 1-2$, Didactiques et sciences de l'ducation, université de Caen - Basse-Normandie, pp. 23-29.

Mercier, Alain, Schubauer-Leoni, Maria-Luisa, Sensevy, Gérard (2002) : "Vers une didactique comparée ", Revue Française de Pédagogie n 141, Vers une didactique comparée, octobre-novembre-décembre 2002, Paris, INRP, pp. 5-16.

PASSERON, Jean-Claude (1991/2006) : Le Raisonnement sociologique, Paris, Nathan.

PAVEAU, Marie-Anne (2001) : « Euvre littéraire et textes journalistiques : la querelle des implicites », Le Français aujourd'hui $\mathrm{n}^{\circ} 134$, Les Textes de presse, Paris, AFEF, juillet 2001, pp. 15-31.

PERrin-Glorian, Marie-Jeanne, Reuter, Yves dir. (2006) : Les Méthodes de recherche en didactiques, Villeneuve d'Ascq, Presses universitaires du Septentrion.

PETITJEAN, André (2001) : «Écriture d'invention et enseignement de la littérature au lycée ? », Enjeux n 51/52, Namur, CEDOCEF, pp. 163-179.

PLANE, Sylvie (2001) : «La réception des nouvelles approches de l'écriture au lycée : le difficile passage de l'écriture du lycéen à l'écriture de l'auteur », Lidil $\mathrm{n}^{\circ} 23$, Les nouveaux écrits à l'école : nouveaux programmes, nouvelles pratiques, nouveaux savoirs, Grenoble, LIDILEM, juillet 2001, pp. 67-82. 
PRATIQUES n 137-138 (2008) : La didactique du français. Hommage à Jean-François Halté, Metz, CRESEF.

PRATIQUES $\mathrm{n}^{\circ} 32$ (1981): La littérature et ses institutions, Metz, CRESEF.

REPÈRES $\mathrm{n}^{\circ} 45$ (en préparation) Vingt ans de recherches en didactique du français années 1990-2010, Lyon, INRP.

REUTER, Yves (1995/2005) : «Didactique du français : éléments de réflexion et de proposition, dans Chiss Jean-Louis, David Jacques, Reuter Yves dir., Didactique du français. Fondements d'une discipline, Bruxelles, De Boeck, pp. 211-231.

- (2004) : «Analyser la discipline : quelques propositions », La Lettre de l'AIRDF n ${ }^{\circ} 35$, Namur, pp. 5-12.

- (2005) : «Définition, statut et valeurs des dysfonctionnements en didactique », Repères $\mathrm{n}^{\circ} 31$, L'Évaluation en didactique du français : résurgence d'une problématique, Paris, INRP, pp. 211-231.

- (2006) : «À propos des usages de Goody en didactique. Éléments d'analyse et de discussion », Pratiques $n^{\circ}$ 131-132, La littératie. Autour de Jack Goody, Metz, CRESEF, pp. 131- 154.

- (2008) : «Didactique du français », dans Van Zanten Agnès dir., Dictionnaire de l'éducation, Paris, PUF, pp. 147-149.

— (à paraître) : «À propos de la notion de performance en didactiques. Éléments de discussion ", Recherches en didactiques. Les Cahiers Théodile $\mathrm{n}^{\circ} 11$, REDLCT, Lille 3.

- dir. (2005) : Pédagogie du projet et didactique du français. Penser et débattre avec Francis Ruellan, Villeneuve d'Ascq, Presses Universitaire du Septentrion.

- dir. (2007a) : Dictionnaire des concepts fondamentaux des didactiques, Bruxelles, De Boeck.

— dir. (2007b) : Une école Freinet. Fonctionnements et effets d'une pédagogie alternative en milieu populaire, Paris, L'Harmattan.

ROSIER, Jean-Maurice (2002) : La didactique du français, Paris, PUF, collection «Que sais-je?».

ROSIER, Jean-Maurice, DUPONT, Didier, REUTER, Yves (1988/2000) : S'approprier le champ littéraire. Propositions pour travailler l'institution littéraire en classe de français, Bruxelles, De Boeck-Duculot.

RUELLAN, Francis (2000): Un mode de travail didactique pour l'enseignement-apprentissage de l'écriture au cycle 3 de l'école primaire, thèse soutenue le 14 janvier 2000 à l'université Charles-de-Gaulle - Lille 3, Lille, ANRT.

SCHNEUWLY, Bernard (1990) : «Didactique : quelques notes sur son histoire », La Lettre de la DFLM n ${ }^{\circ}$, Paris, DFLM, pp. 22-24.

VERRET, Michel (1975): Le Temps des études, Paris, Librairie Honoré Champion.

VINCENT, Guy (1980) : L'école primaire française, Lyon, Presses universitaires de Lyon. 